\title{
The factors contributing to cognitive dysfunction in type 2 diabetic patients
}

\author{
San-Shan Xia, Wen-Lin Xia, Jiao-Jiao Huang, Hua-Jie Zou, Jing Tao, Yan Yang \\ Department of Endocrinology, Tongji Hospital, Tongji Medical College, Huazhong University of Science and Technology, Wuhan 430030, China \\ Contributions: (I) Conception and design: SS Xia, Y Yang; (II) Administrative support: J Tao; (III) Provision of study materials or patients: SS Xia, \\ WL Xia; (IV) Collection and assembly of data: SS Xia, JJ Huang; (V) Data analysis and interpretation: SS Xia, HJ Zou; (VI) Manuscript writing: All \\ authors; (VII) Final approval of manuscript: All authors. \\ Correspondence to: Yan Yang. Department of Endocrinology, Tongji Hospital, Tongji Medical College, Huazhong University of Science and \\ Technology, Wuhan 430030, China. Email: yangyan6910@163.com.
}

\begin{abstract}
Backgrounds: The aim of the research was to investigate the factors contributing to cognitive dysfunction in type 2 diabetic patients, to distinguish the complex relationship between diabetic retinopathy (DR) and different cognitive status.
\end{abstract}

Methods: Two hundred and ninety-seven type 2 diabetes mellitus (T2DM) patients were enrolled in our study. We adopted the Clinical Dementia Rating (CDR), Mini-mental State Examination (MMSE) and Montreal Cognitive Assessment (MOCA) to evaluate the cognitive function. Firstly, cognition status was classified into dementia and non-dementia according to MMSE and CDR. Patients with non-dementia were further classified into mild cognitive impairment (MCI) and normal cognition status based on MOCA. The factors contributing to cognitive dysfunction were analyzed.

Results: Among the 297 T2DM subjects, 47 were enrolled in the dementia group and 174 in the MCI group according to a battery of cognitive function tests, presenting a prevalence of $15.8 \%$ and $58.6 \%$ respectively. After adjustment for age, sex, and education level, waist circumference and DR were risk factors for dementia (OR: 1.057, P=0.011; OR: 2.197, P=0.040). Low-density lipoprotein cholesterol (LDL-C) was a risk factor for MCI (OR: 1.635, $\mathrm{P}=0.047)$, while age at $\mathrm{T} 2 \mathrm{DM}$ onset and moderate drinking were protective factors for MCI (OR: 0.936, P=0.044; OR: 0.289, P=0.004).

Conclusions: MCI is common in T2DM patients. Waist circumference and DR are risk factors of dementia, LDL-C is a risk factor for MCI, and moderate drinking and age at T2DM onset are protective factors for MCI. DR is unrelated to MCI in T2DM.

Keywords: Type 2 diabetes mellitus (T2DM); dementia; mild cognitive impairment (MCI); diabetic retinopathy (DR)

Submitted Sep 21, 2019. Accepted for publication Dec 06, 2019.

doi: 10.21037/atm.2019.12.113

View this article at: http://dx.doi.org/10.21037/atm.2019.12.113

\section{Introduction}

As familiar chronic diseases, type 2 diabetes mellitus (T2DM), as well as Alzheimer's disease-related dementias (ADRDs), have a major impact on the quality of life of the ageing populations (1). Epidemiological studies have shown that T2DM increases the risk of ADRDs by 1.5-2.5 times compared to age-matched non-diabetic subjects $(2,3)$. Interestingly, T2DM and ADRDs shared a wide range of pathophysiological mechanisms including oxidative stress, amyloidosis, aberrant enzymatic activity, endothelial dysfunction, brain insulin resistance and deficiency, and even shared genetic background (4). Therefore, some studies suggested that ADRDs may be type 3 diabetes mellitus (T3DM) (5).

Mild cognitive impairment (MCI) is the early stage of dementia, defined as a slight cognitive dysfunction which is noticeable and measurable decline in cognitive abilities assessment, but not impact the daily living yet (6). The 
annual conversion rate of MCI to dementia ranges from $5-20 \%$ in the general population (7), and it accelerates under the pathologic conditions of T2DM (8). In this regard, early screening and intervention of MCI patients in time is of great importance for reducing the occurrence of dementia in T2DM.

In addition, T2DM patients would be more vulnerable to cognitive impairment than control group, presenting a damaged self-management of diabetes (9). In clinical practice, there are few reliable phenotypic indicators to determine whether T2DM patients are at risk of developing dementia. As the most specific microvascular complication of diabetes, diabetic retinopathy (DR) shares an overlapped pathophysiology with cognitive decline (10). Several studies have explored the association of DR with cognitive impairment, however, their conclusions were conflicting $(11,12)$. Thus, our aims are to investigate the risk factors of cognitive dysfunction in T2DM patients with MCI and dementia, especially in the consideration of DR.

\section{Methods}

\section{Study design and participants}

In the Department of Endocrinology, Tongji Hospital, Tongji Medical College, Huazhong University of Science and Technology, from April 2018 to May 2019, we assessed the cognition of T2DM inpatients according to T2DM Diagnostic Standards from the American Diabetes Association (13): fasting plasma glucose (FPG) $\geq 7.0 \mathrm{mmol} / \mathrm{L}$ and/or postprandial glucose (PPG) $\geq 11.1 \mathrm{mmol} / \mathrm{L}$, or in a patient with classic symptoms of hyperglycemia or hyperglycemic crisis, a random plasma glucose $\geq 11.1 \mathrm{mmol} / \mathrm{L}$, or hemoglobin A1c (HbA1c) $\geq 6.5 \%$, and clearly classified as type 2 . Core Clinical Criteria for Dementia Diagnostics from the National Institute of Aging and Alzheimer's Association (NIA-AA) (14) and the Diagnostic and Statistical Manual of Mental Disorders, $4^{\text {th }}$ edition (DSM-IV) (15), the essential criteria for dementia included: (I) represent an interference with the ability to function at occupational or social activities; and (II) represent a decline from previous levels of memory and at least one area of performance and functioning is impaired; and (III) the decline in function can not be explained by neurological explanations such as delirium or other psychiatric disorder. We didn't perform the dementia typing in this study. Core clinical criteria for MCI of from NIA-AA (16) and Petersen's criteria (17) include: (I) concern in terms of a change in cognitive performance; and (II) impairment in at least one cognitive domain; and (III) preservation of independence in social functional abilities; and (IV) not demented. Therefore, a comprehensive battery of cognitive tests which was internationally recognized were applied, including the Clinical Dementia Rating (CDR), Mini-Mental State Examination (MMSE) and Montreal Cognitive Assessment (MOCA). We took a general cut point of those screening tests, which had been suggested by a large body of literature. Lower CDR score means better cognitive performance, CDR score was bounded by $1.0 / 0.5$ (score considered "positive"/"negative") for dementia, 0.5/0 for MCI (18). Higher values of MMSE and MOCA evaluations indicate better cognition, MMSE score was bounded by 23/24 for dementia (19), and MOCA score by 25/26 for MCI (20), meanwhile, among the participants who had 12 years of education or fewer, a point was added to his/ her total MOCA score to calibrate the bias of education level (21). Synthesis score of CDR was calculated online (http://www.biostat.wustl.edu/ adrc/cdrpgm/index.html). A neurologist who was qualified after the memory clinic training performed the cognitive assessment and verified the diagnosis of MCI and dementia.

\section{Inclusion and exclusion criteria}

Inclusion criteria: (I) T2DM met the ADA diagnostic criteria (see Method section for details); (II) age: 45-74 years old; (III) education level: low (6-12 years) or high ( $>12$ years); (IV) the clinical manifestations of memory deficit, and confirmed by others; (V) had the visual and auditory ability to take neuropsychological test.

Exclusion criteria: (I) other types of diabetes; (II) younger than 45-year-old or older than 75; (III) less than 6 years of education; (IV) patients who were not able to complete neuropsychological scale screening, such as speech impairment, hearing loss and reluctant to cooperate then; (V) combined with metabolic diseases that may affect cognitive function temporarily, such as acute carbohydrate metabolic events in the last 3 months, including severe hypoglycemia, diabetic ketoacidosis, diabetic hyperglycemia hypertonic coma, hypothyroidism, etc.; (VI) combined with head trauma that might affect cognitive function, cerebral intake, mental and neurological disorders such as depression, anxiety, delirium, severe lung or kidney diseases, history of heart failure, malignant tumors, etc.; (VII) a history of drug dependence/abuse; (VIII) used the following 
drugs in the previous month: antidepressant drugs, antiParkinson drugs, anti-epileptic drugs, sedative hypnotic drugs, etc.; (IX) using cognitive dysfunction medications such as donepezil, memantine, etc.

\section{Measurements}

After an overnight fasting, we accomplished the subject's laboratory inspection including FPG, fasting plasma C-peptide (FC-P), diabetes complication screening, completed self-monitoring of blood glucose (SMBG) confirmed by the glucose oxidase method (22). Glycemic control was characterized by HbAlc, postprandial glucose excursion (PPGE), standard deviation of blood glucose (SDBG), and largest amplitude of glycemic excursions (LAGE). Diabetic macrovascular complications were defined as: peripheral artery disease, transient ischemic attack, stroke, coronary heart disease and heart failure. Homeostasis model assessment 2 of $\beta$-cell function (HOMA2- $\beta$ ) and Homeostasis model assessment 2 of Insulin Resistance index (HOMA2-IR) were produced by homeostasis model calculator to evaluate insulin function (Windows v2.2.3 version ${ }^{\odot}$ Diabetes Trials Unit, University of Oxford, http://www.dtu.ox.ac.uk/homa, visited May 20, 2019) (23).

Covariates included demographic characteristics, cardiovascular-related behaviors, and chronic diseases were analyzed; Demographic characteristics were age, sex, and education level; cardiovascular-related behaviors were smoking (current smoker and ex-smoker, or never smoked), alcohol consumption per day [abstainer (zero grams), moderate drinker (less than $60 \mathrm{~g}$ for men, 40 for women); chronic disease covariates were based on self-report of medical diagnosis or confirmed by medical records], including hypertension (defined as blood pressure of $140 / 90 \mathrm{mmHg}$ or higher, or use of antihypertensive drugs) (24), obesity [defined as body mass index (BMI) $\geq 28 \mathrm{~kg} / \mathrm{m}^{2}$ ].

\section{Statistical analysis}

The data analysis was carried out in SPSS version 24.0 for Windows. We did cross-sectional analyses of cognition status by Student's $t$-test (parametric) or Kruskal-Wallis test (nonparametric) for continuous data and $\chi^{2}$ tests for categorical data. Logistic regression analysis was conducted to predict dementia $v s$. non-dementia and MCI $v s$. normal cognition status, performed by using the following variables: sociodemographic measures, health-related behaviors, vascular risk factors and diabetic-specific parameters. Variance inflation factor (VIF) value was used to evaluate multicollinearity. $\mathrm{P}$ values were two sided and $\mathrm{P}<0.05$ was regarded as significant.

\section{Results}

\section{Two bundred and ninety-seven participants were finally involved in our study}

Figure 1 described the flow chart of our study. Among the 591 subjects who were diagnosed as T2DM, 297 subjects were finally enrolled in the study according to the inclusion criteria. Cross-sectional analyses showed that the average age was 56.8 [standard deviation (SD): 6.9] years, of which 47 (15.8\%) had dementia, and 174 (58.6\%) had MCI. Subjects who failed to participate in had lower education level than subjects in our study $(85.0 \%$ vs. $77.4 \%, \mathrm{P}<0.05)$; however, they were similar in age, sex, and history of hypertension.

\section{The factors contributing to dementia}

Table 1 presents the defining characteristics of the study population by cognition status between dementia and non-dementia. Compared with non-dementia individuals, women $(51.1 \%$ vs. $32.4 \%)$, the percentage of low education $(85.6 \%$ to $63.2 \%)$ in dementia group were increased significantly $(\mathrm{P}<0.05)$. There were no significant differences in blood pressure, lipids, course, glycemic excursion, the function of islet cells and the prevalence of diabetes complications.

After adjustment for age, sex and education level, those with DR had 2.197 times (95\% CI: 1.035-4.664) higher risk for dementia than those without DR (Table 2). Meanwhile, the waist circumference $(\mathrm{OR}: 1.057, \mathrm{P}=0.011)$ was a risk factor for dementia. DR was the most significant element contributing to dementia.

\section{The factors contributing to MCI}

Table 3 shows the characteristics of the study population by cognition status between MCI and normal cognition. The mean duration of diabetes in subjects with MCI was 8.7 (SD: 6.5 ) years, the HbA1c was $8.8 \%$ (SD: $2.1 \%)$. Compared with normal cognition individuals, the age (57.1 vs. 55.0 years), women (37.4\% vs. $21.1 \%$ ), low education level ( $85.6 \%$ vs. $63.2 \%$ ), high-density 


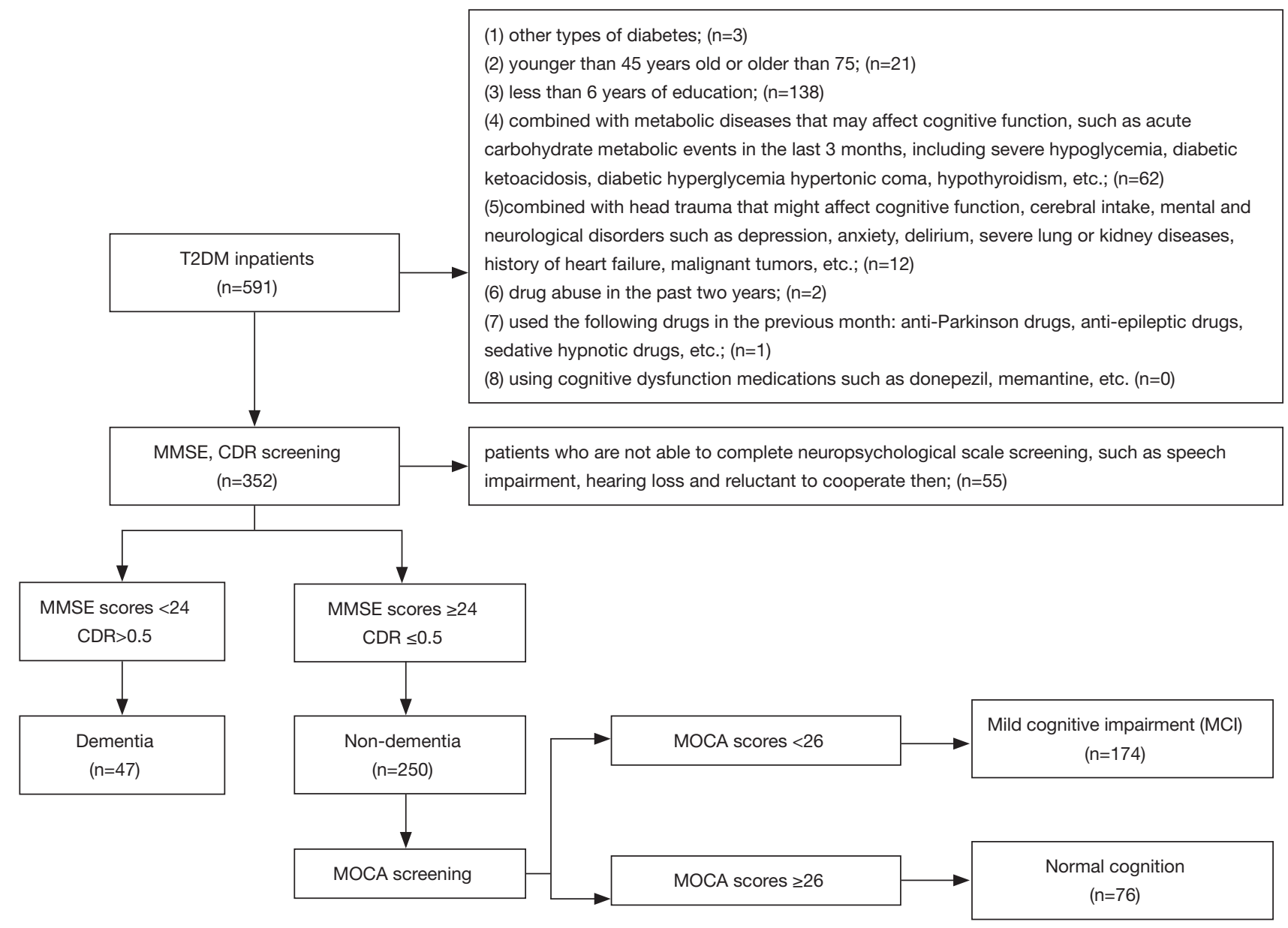

Figure 1 Flow chat of study participants: 47 had dementia, 174 had MCI, and 76 had normal cognition. Exclusion criteria had shown above. MCI, mild cognitive impairment; T2DM, type 2 diabetes mellitus; MMSE, Mini-mental State Examination; CDR, Clinical Dementia Rating; MOCA, Montreal Cognitive Assessment.

lipoprotein cholesterol (HDL-C) (1.05 vs. $0.96 \mathrm{mmol} / \mathrm{L})$, low-density lipoprotein cholesterol (LDL-C) (2.59 vs. $2.34 \mathrm{mmol} / \mathrm{L})$ in MCI group increased significantly $(\mathrm{P}<0.05)$, waist circumference $(90.2$ vs. $93.1 \mathrm{~cm})$ strikingly decreased, while there were no significant differences in the course, glycemic excursion, islet cell function, and the prevalence of diabetes complications.

After adjustment for age, sex and education level, the results showed that LDL-C (OR: $1.635, \mathrm{P}=0.047)$ was a risk factor for MCI, the age at T2DM onset (OR: 0.936, $\mathrm{P}=0.044)$, moderate drinking (OR: $0.289, \mathrm{P}=0.004)$ was the protective factor of MCI, and moderate drinking was the most significant factor affecting MCI (Table 4). But the results for the diabetes parameters such as $\mathrm{HbA1c}$, course and DR were not significant.

\section{Discussion}

Cognitive performance is based on brain function, which relies heavily on energy supply and is regulated by physiological parameters such as hormones (25). Insulin resistance and hyperinsulinemia are common in T2DM. Our hypothesis is that there are reliable phenotypic indicators to forecast whether T2DM patients are at risk of developing dementia, especially in the consideration of DR. Our results showed that DR had an increased risk of dementia, but seemed to have no relation with MCI.

Among T2DM subjects, the prevalence of dementia was about $15.8 \%$, the prevalence of MCI was about $58.6 \%$, and the rate of cognitive dysfunction was almost 2 times higher than that of healthy people, which was consistent with previous studies (26). Our results also showed that 
Table 1 Characteristics of the study population by cognition status

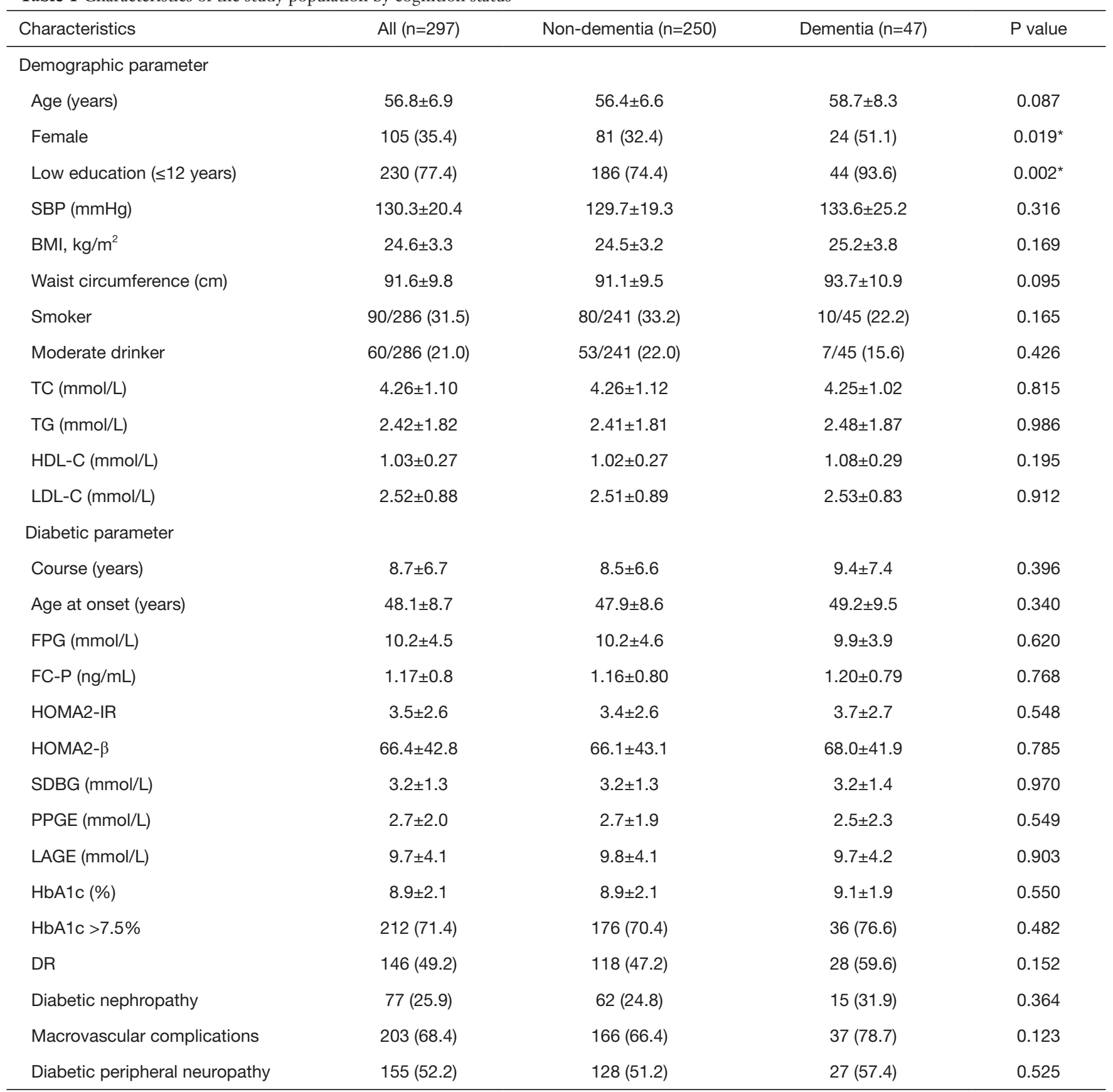

Data are displayed in the form of $\mathrm{n}(\%)$ or mean \pm SD. ${ }^{*}$, There were significant differences between non-dementia group and dementia group. SBP, systolic blood pressure; BMI, body mass index; TC, total cholesterol; TG, triglyceride; LDL-C, low-density lipoprotein cholesterol; HDL-C, high-density lipoprotein cholesterol; FPG, fasting plasma glucose; FC-P, fasting plasma C-peptide; HOMA2-IR, homeostasis model assessment 2 of insulin resistance index; HOMA2- $\beta$, homeostasis model assessment 2 of $\beta$-cell function; SMBG, selfmonitoring of blood glucose; SDBG, standard deviation of blood glucose; PPGE, postprandial glucose excursion; LAGE, largest amplitude of glycemic excursions; HbA1c, hemoglobin A1c; DR, diabetic retinopathy. 
Table 2 Logistic regression analysis for the risks of dementia

\begin{tabular}{lcc}
\hline Variables & OR & 95\% Cl \\
\hline DR & 2.197 & $1.035-4.664$ \\
Waist circumference $(\mathrm{cm})$ & 1.057 & $1.013-1.103$ \\
LAGE & 0.909 & $0.818-1.009$ \\
Constant term & 0.000 & - \\
\hline
\end{tabular}

ORs (95\% Cls) were adjusted for age, sex and education level. DR, diabetic retinopathy; LAGE, largest amplitude of glycemic excursions.

moderate alcohol consumption was a protective factor for MCI, LDL-C was a risk factor for MCI, DR and waist circumference were risk factors for dementia. We recorded little attenuation of associations after taking potential confounding factors into account. Several studies have suggested that merely the elderly people with T2DM produce an effect on faster rate of cognitive decline, with the hypothesis that T2DM does not affect cognition before old age (18), however, our results showed that MCI affected more than half of our T2DM participants, most of which were in their fifties. This result was in line with previous findings, suggesting that more attention should be paid to the prevention and control of dementia in middle-age rather than twilight years in T2DM patients (24).

Consistent with our research, previous high-quality studies have shown that in elderly women with T2DM, high waist circumference was associated with decreased cognitive domains such as language and executive ability, and overall cognitive performance (27). At present, the relationship between LDL-C and cognitive dysfunction is confusing (28), the use of lipid-lowering drugs does not improve late T2DM cognitive function, too (29). The results of our study indicated that LDL-C was one of the risk factors for MCI. The fundamental reason may be that LDL-C increase risk of atherosclerosis, affecting blood circulation in the brain.

Moderate drinking has potential benefits for cognitive performance, Alzheimer's disease and other dementias, with unknown mechanisms $(30,31)$. The relationship of alcohol consumption with cognitive outcomes is thought to be J-shaped or U-shaped, which means both abstinence and excessive alcohol consumption have harmful effects on cognition, while moderate alcohol consumption appears to lower the risk of dementia (32-34), our research support the hypothesis. Some researchers have investigated the underlying mechanism of this phenomenon, they claimed a mediating role of cardiometabolic disease between moderate alcohol consumption and dementia, some of the excess risk of dementia in abstainers may be explained by greater risk of cardiometabolic disease in this group $(35,36)$. Moderate drinkers tended to have a healthy lifestyle, they rarely smoke, and take a healthy, balanced diet. Furthermore, moderate drinkers were more likely to drink wine, while excessive drinkers were more likely to drink beer (37). Of course, the result may be influenced by other factors, it is not an easy task to investigate the association between alcohol consumption and cognitive impairment, because this task is fraught with methodological difficulties (38). Moreover, given the negative effects of alcohol intake on diabetes (39), we cannot simply encourage or discourage moderate drinking in T2DM patients.

There is little research on relationships about the age at T2DM onset and cognitive dysfunction at an advanced stage. Compared to age and diabetes course, the age at T2DM onset is a classical and historical factor which has been used to identify the diversity of diabetes (40). Recently, studies center on the new classification of diabetes have shown that the age at T2DM onset was the main factor in distinguishing different types of diabetes (41), and as a branch of T2DM, mild age-related diabetes (MARD) patients had significantly higher age at T2DM onset and milder clinical symptoms compared with other groups: their glucose or lipid metabolism disorders, insulin resistance were not obvious, and autoimmune antibodies were negative, in line with the previous study $(42,43)$. Our results showed that the higher age at T2DM onset was a protective factor of MCI, suggesting that MARD subtype may be related to milder cognitive impairment than other subtypes. Further clinical studies could explore the relationship between new types of diabetes and cognitive dysfunction.

Our results found that the peripheral insulin resistance index represented by HOMA2-IR was not related to cognitive performance in T2DM subjects. The results are consistent with the latest large-scale prospective cohort study (44). Hyperinsulinemia caused by insulin resistance depletes peripheral insulin-degrading enzymes that affect the 
Table 3 Characteristics of the Non-dementia group by cognition status between MCI and normal cognition

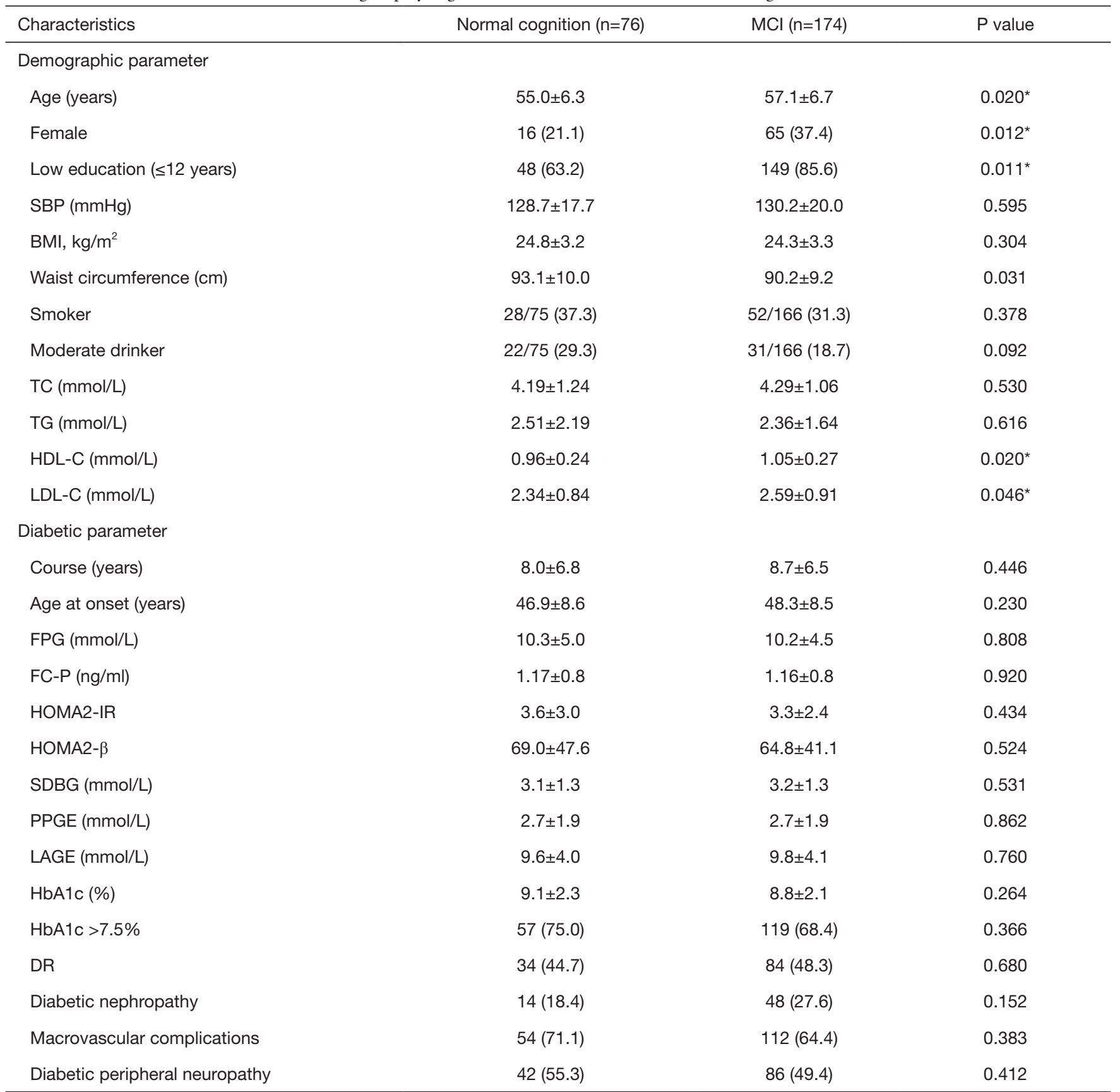

Data are displayed in the form of $\mathrm{n}(\%)$ or mean (SD). *, There were significant differences between normal cognition group and MCl group. $\mathrm{MCl}$, mild cognitive impairment; SBP, systolic blood pressure; BMI, body mass index; TC, total cholesterol; TG, triglyceride; LDL-C, lowdensity lipoprotein cholesterol; HDL-C, high-density lipoprotein cholesterol; FPG, fasting plasma glucose; FC-P, fasting plasma C-peptide; HOMA2-IR, homeostasis model assessment 2 of insulin resistance index; HOMA2- $\beta$, homeostasis model assessment 2 of $\beta$-cell function; SMBG, self-monitoring of blood glucose; SDBG, standard deviation of blood glucose; PPGE, postprandial glucose excursion; LAGE, largest amplitude of glycemic excursions; HbA1c, hemoglobin A1c; DR, diabetic retinopathy. 
Table 4 Logistic regression analysis for the risks of MCI

\begin{tabular}{lcc}
\hline Varieties & OR & $95 \% \mathrm{Cl}$ \\
\hline LDL-C & 1.635 & $1.006-2.656$ \\
Age at T2DM onset & 0.936 & $0.878-0.998$ \\
Diabetic peripheral neuropathy & 0.463 & $0.196-1.096$ \\
Moderate drinker & 0.289 & $0.124-0.674$ \\
Constant term & 0.005 & - \\
\hline
\end{tabular}

ORs (95\% Cls) were adjusted for age, sex and education level. MCl, mild cognitive impairment; LDL-C, low density lipoprotein cholesterol; T2DM, type 2 diabetes mellitus.

metabolism of $\beta$-amyloid protein (A $\beta)$, which causes a buildup of A $\beta$. But, HOMA2-IR can only reflect insulin resistance of peripheral effectors such as liver and skeletal muscles. Previous studies have shown that brain insulin resistance was closely related to cognitive dysfunction (1), so there is in need of further clinical research about insulin resistance.

Numerous clinical studies have presented a nonlinear association between the glycemic control and risk of dementia (45). On the one hand, the cognitive function of patients with glucose peaks decreased significantly compared with the control group (46). Hyperglycemia might affect cognitive function through injury to vascular endothelial cells, swelling of metabolites, or aggravation of oxidative stress. On the other hand, repeated episodes of hypoglycemia caused by enhanced hypoglycemic methods significantly impaired cognitive function in T2DM patients (47). Thus, no matter what situation carbohydrate metabolism disorders is in, it may increase the risk of dementia in T2DM. In our study, there was no significant relationship among HbA1c, glycemic excursion and cognitive dysfunction, suggesting that carbohydrate metabolism may be too complex to be an appropriate indicator for the cognitive dysfunction in T2DM.

Our study shows DR is a risk factor for dementia which is consistent with previous studies (48). The researchers have found that brain functional networks were altered in DR patients, which specifically reflected the severity of microvascular injury and visual acuity weakness. DR might affect cognition-related functional networks through changes in the visual function (49). Further studies showed that there were neurodegenerative changes in the retina in patients with DR, meanwhile the retinal vascular damage and neurodegenerative lesions were associated with cognitive dysfunction. The correlation between DR and cognitive dysfunction was likely to be mediated by diabetic specific vascular injury and retinal neurodegeneration (10). However, our results showed that DR is unrelated to MCI which is the early stage of ADRDs and hard for clinician to find it out, it may be too late to intervene cognitive dysfunction in T2DM patients when they have had the DR. These findings suggest that DR contributes to faster develop to dementia before the clinician could recognize, and that we should prevent the DR to control the risk of cognitive decline. The next step is to design a prospective cohort study to verify our hypothesis.

The strengths of this analysis are the relatively young population- $71 \%$ of our participants were younger than 60 years at the cognitive assessment. We discuss about cognitive dysfunction in T2DM with a view to MCI, it is the inevitable stage of ADRDs (50) but neglected in clinical work. We also allow for a range of potential confounders that combine diabetes with cognitive function. The main limitation of our study seems to be the aspect of generalizability, since the data comes from inpatients in Tongji hospital and the participants are likely to be more seriously ill than the general population in the view of cognitive dysfunction.

\section{Conclusions}

Our results support the hypothesis that DR may predict the risk of dementia in T2DM, but it is too distant a signal for MCI. The age at T2DM onset is an important protective factor for MCI. Further cohort studies and research on mechanisms are required to identify whether improving prevention of DR or screening early-onset T2DM patients would reduce the risk of cognitive dysfunction.

\section{Acknowledgments}

We would like to thank all volunteers who participate in 
this study enthusiastically.

Funding: This work was supported by the National Natural Science Foundation of China (grant number 81670754).

\section{Footnote}

Conflict of Interest: The authors have no conflicts of interest to declare.

Ethical Statement: The authors are accountable for all aspects of the work in ensuring that questions related to the accuracy or integrity of any part of the work are appropriately investigated and resolved. This study protocol has been approved by the Medical Ethics Committee of Tongji Hospital (No. TJ-C20160206), and all subjects have signed an informed consent, in line with the Declaration of Helsinki as revised in 2013.

\section{References}

1. Arnold SE, Arvanitakis Z, Macauley-Rambach SL, et al. Brain insulin resistance in type 2 diabetes and Alzheimer disease: concepts and conundrums. Nat Rev Neurol 2018;14:168-81.

2. Zhang J, Chen C, Hua S, et al. An updated meta-analysis of cohort studies: diabetes and risk of Alzheimer's disease. Diabetes Res Clin Pract 2017;124:41-7.

3. Xu W, Caracciolo B, Wang HX, et al. Accelerated progression from mild cognitive impairment to dementia in people with diabetes. Diabetes 2010;59:2928-35.

4. de Matos AM, de Macedo MP, Rauter AP. Bridging type 2 diabetes and Alzheimer's disease: assembling the puzzle pieces in the quest for the molecules with therapeutic and preventive potential. Med Res Rev 2018;38:261-324.

5. de la Monte SM. The full spectrum of Alzheimer's disease is rooted in metabolic derangements that drive type 3 diabetes. Adv Exp Med Biol 2019;1128:45-83.

6. Petersen RC, Lopez O, Armstrong MJ, et al. Practice guideline update summary: mild cognitive impairment: report of the Guideline Development, Dissemination, and Implementation Subcommittee of the American Academy of Neurology. Neurology 2018;90:126-35.

7. Langa KM, Levine DA. The diagnosis and management of mild cognitive impairment: a clinical review. JAMA 2014;312:2551-61.

8. Yuan XY, Wang XG. Mild cognitive impairment in type 2 diabetes mellitus and related risk factors: a review. Rev Neurosci 2017;28:715-23.
9. Munshi MN. Cognitive dysfunction in older adults with diabetes: what a clinician needs to know. Diabetes Care 2017;40:461-7.

10. Simó R, Stitt AW, Gardner TW. Neurodegeneration in diabetic retinopathy: does it really matter? Diabetologia 2018;61:1902-12.

11. Ding J, Strachan MW, Reynolds RM, et al. Diabetic retinopathy and cognitive decline in older people with type 2 diabetes: the Edinburgh Type 2 Diabetes Study. Diabetes 2010;59:2883-9.

12. Crosby-Nwaobi RR, Sivaprasad S, Amiel S, et al. The relationship between diabetic retinopathy and cognitive impairment. Diabetes Care 2013;36:3177-86.

13. American Diabetes Association. Standards of medical care in diabetes--2010. Diabetes Care 2010;33 Suppl 1:S11-61.

14. McKhann GM, Knopman DS, Chertkow H, et al. The diagnosis of dementia due to Alzheimer's disease: recommendations from the National Institute on AgingAlzheimer's Association workgroups on diagnostic guidelines for Alzheimer's disease. Alzheimers Dement 2011;7:263-9.

15. American Psychiatric Association. Diagnostic and Statistical Manual of Mental Disorders. 4th ed. Washington DC: American Psychiatric Association, 1994.

16. Albert MS, DeKosky ST, Dickson D, et al. The diagnosis of mild cognitive impairment due to Alzheimer's disease: recommendations from the National Institute on AgingAlzheimer's Association workgroups on diagnostic guidelines for Alzheimer's disease. Alzheimers Dement 2011;7:270-9.

17. Petersen RC. Mild cognitive impairment as a diagnostic entity. J Intern Med 2004;256:183-94.

18. Roberts RO, Knopman DS, Geda YE, et al. Association of diabetes with amnestic and nonamnestic mild cognitive impairment. Alzheimers Dement 2014;10:18-26.

19. Moyer VA, Force USPST. Screening for cognitive impairment in older adults: U.S. Preventive Services Task Force recommendation statement. Ann Intern Med 2014;160:791-7.

20. Tsoi KK, Chan JY, Hirai HW, et al. Cognitive tests to detect dementia: a systematic review and meta-analysis. JAMA Intern Med 2015;175:1450-8.

21. Nasreddine ZS, Phillips NA, Bedirian V, et al. The Montreal Cognitive Assessment, MoCA: a brief screening tool for mild cognitive impairment. J Am Geriatr Soc 2005;53:695-9.

22. Tabák AG, Jokela M, Akbaraly TN, et al. Trajectories of glycaemia, insulin sensitivity, and insulin secretion before 
diagnosis of type 2 diabetes: an analysis from the Whitehall II study. Lancet 2009;373:2215-21.

23. Zou X, Zhou X, Zhu Z, et al. Novel subgroups of patients with adult-onset diabetes in Chinese and US populations. Lancet Diabetes Endocrinol 2019;7:9-11.

24. Tuligenga RH, Dugravot A, Tabák AG, et al. Midlife type 2 diabetes and poor glycaemic control as risk factors for cognitive decline in early old age: a post-hoc analysis of the Whitehall II cohort study. The Lancet Diabetes \& Endocrinology 2014;2:228-35.

25. Butterfield DA, Halliwell B. Oxidative stress, dysfunctional glucose metabolism and Alzheimer disease. Nat Rev Neurosci 2019;20:148-60.

26. Chan KY, Wang W, Wu JJ, et al. Epidemiology of Alzheimer's disease and other forms of dementia in China, 1990-2010: a systematic review and analysis. Lancet 2013;381:2016-23.

27. West RK, Ravona-Springer R, Heymann A, et al. Waist circumference is correlated with poorer cognition in elderly type 2 diabetes women. Alzheimers Dement 2016;12:925-9.

28. Feinkohl I, Price JF, Strachan MW, et al. The impact of diabetes on cognitive decline: potential vascular, metabolic, and psychosocial risk factors. Alzheimers Res Ther 2015;7:46.

29. Williamson JD, Launer LJ, Bryan RN, et al. Cognitive function and brain structure in persons with type 2 diabetes mellitus after intensive lowering of blood pressure and lipid levels: a randomized clinical trial. JAMA Intern Med 2014;174:324-33.

30. Collins MA, Neafsey EJ, Mukamal KJ, et al. Alcohol in moderation, cardioprotection, and neuroprotection: epidemiological considerations and mechanistic studies. Alcohol Clin Exp Res 2009;33:206-19.

31. Verbaten MN. Chronic effects of low to moderate alcohol consumption on structural and functional properties of the brain: beneficial or not? Hum Psychopharmacol 2009;24:199-205.

32. Anstey KJ, Mack HA, Cherbuin N. Alcohol consumption as a risk factor for dementia and cognitive decline: metaanalysis of prospective studies. Am J Geriatr Psychiatry 2009;17:542-55.

33. Panza F, Frisardi V, Seripa D, et al. Alcohol consumption in mild cognitive impairment and dementia: harmful or neuroprotective? Int J Geriatr Psychiatry 2012;27:1218-38.

34. Xu W, Wang H, Wan Y, et al. Alcohol consumption and dementia risk: a dose-response meta-analysis of prospective studies. Eur J Epidemiol 2017;32:31-42.

35. Li R, Singh M. Sex differences in cognitive impairment and Alzheimer's disease. Front Neuroendocrinol 2014;35:385-403.

36. de Bruijn RF, Ikram MA. Cardiovascular risk factors and future risk of Alzheimer's disease. BMC Med 2014;12:130.

37. Sabia S, Fayosse A, Dumurgier J, et al. Alcohol consumption and risk of dementia: 23 year follow-up of Whitehall II cohort study. BMJ 2018;362:k2927.

38. Rao RT. Methodological difficulties of studying alcohol consumption and dementia. BMJ 2018;362:k3894.

39. Rehm J, Gmel GE, Sr., Gmel G, et al. The relationship between different dimensions of alcohol use and the burden of disease-an update. Addiction 2017;112:968-1001.

40. Leslie RD, Palmer J, Schloot NC, et al. Diabetes at the crossroads: relevance of disease classification to pathophysiology and treatment. Diabetologia 2016;59:13-20.

41. Ahlqvist E, Storm P, Käräjämäki A, et al. Novel subgroups of adult-onset diabetes and their association with outcomes: a data-driven cluster analysis of six variables. The Lancet Diabetes \& Endocrinology 2018;6:361-9.

42. Al-Saeed AH, Constantino MI, Molyneaux L, et al. An inverse relationship between age of type 2 diabetes onset and complication risk and mortality: the impact of youthonset type 2 diabetes. Diabetes Care 2016;39:823-9.

43. Huo X, Gao L, Guo L, et al. Risk of non-fatal cardiovascular diseases in early-onset versus late-onset type 2 diabetes in China: a cross-sectional study. The Lancet Diabetes \& Endocrinology 2016;4:115-24.

44. Geijselaers SLC, Sep SJS, Claessens D, et al. The role of hyperglycemia, insulin resistance, and blood pressure in diabetes-associated differences in cognitive performancethe Maastricht study. Diabetes Care 2017;40:1537-47.

45. Crane PK, Walker R, Hubbard RA, et al. Glucose levels and risk of dementia. N Engl J Med 2013;369:540-8.

46. Rawlings AM, Sharrett AR, Mosley TH, et al. Glucose peaks and the risk of dementia and 20-year cognitive decline. Diabetes Care 2017;40:879-86.

47. Geijselaers SLC, Sep SJS, Stehouwer CDA, et al. Glucose regulation, cognition, and brain MRI in type 2 diabetes: a systematic review. The Lancet Diabetes \& Endocrinology 2015;3:75-89.

48. Lee CS, Larson EB, Gibbons LE, et al. Associations between recent and established ophthalmic conditions and risk of Alzheimer's disease. Alzheimers Dement 
2019;15:34-41.

49. Dai H, Zhang Y, Lai L, et al. Brain functional networks: correlation analysis with clinical indexes in patients with diabetic retinopathy. Neuroradiology 2017;59:1121-31.
50. Cummings J. The National Institute on Aging-Alzheimer's Association Framework on Alzheimer's disease: application to clinical trials. Alzheimers Dement 2019;15:172-8.
Cite this article as: Xia SS, Xia WL, Huang JJ, Zou HJ, Tao J, Yang Y. The factors contributing to cognitive dysfunction in type 2 diabetic patients. Ann Transl Med 2020;8(4):104. doi: 10.21037/atm.2019.12.113 